\title{
A MT-TL1 variant identified by whole exome sequencing in an individual with intellectual disability, epilepsy, and spastic tetraparesis
}

\author{
Elke de Boer ${ }^{1} \cdot$ Charlotte W. Ockeloen $\mathbb{1}^{2} \cdot$ Leslie Matalonga ${ }^{3} \cdot$ Rita Horvath $\mathbb{D}^{4} \cdot$ Solve-RD SNV-indel working

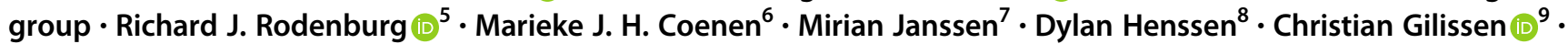

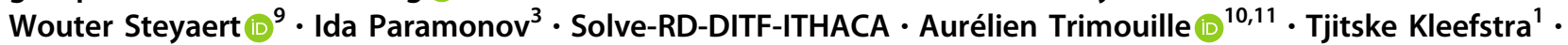 \\ Alain Verloes $\mathbb{1}^{12} \cdot$ Lisenka E. L. M. Vissers $\mathbb{B}^{1}$
}

Received: 13 October 2020 / Revised: 25 March 2021 / Accepted: 15 April 2021 / Published online: 1 June 2021

(c) The Author(s) 2021. This article is published with open access

\begin{abstract}
The genetic etiology of intellectual disability remains elusive in almost half of all affected individuals. Within the Solve-RD consortium, systematic re-analysis of whole exome sequencing (WES) data from unresolved cases with (syndromic) intellectual disability ( $n=1,472$ probands) was performed. This re-analysis included variant calling of mitochondrial DNA (mtDNA) variants, although mtDNA is not specifically targeted in WES. We identified a functionally relevant mtDNA variant in MT-TL1 (NC_012920.1:m.3291T > C; NC_012920.1:n.62T > C), at a heteroplasmy level of 22\% in whole blood, in a 23-year-old male with severe intellectual disability, epilepsy, episodic headaches with emesis, spastic tetraparesis, brain abnormalities, and feeding difficulties. Targeted validation in blood and urine supported pathogenicity, with heteroplasmy levels of $23 \%$ and $58 \%$ in index, and $4 \%$ and $17 \%$ in mother, respectively. Interestingly, not all phenotypic features observed in the index have been previously linked to this MT-TL1 variant, suggesting either broadening of the m.3291T > C-associated phenotype, or presence of a co-occurring disorder. Hence, our case highlights the importance of underappreciated mtDNA variants identifiable from WES data, especially for cases with atypical mitochondrial phenotypes and their relatives in the maternal line.
\end{abstract}

\section{Introduction}

The introduction of whole exome sequencing (WES) in clinical settings has massively augmented diagnostic yield for intellectual disability (ID) and other neurodevelopmental disorders (NDD), and additionally identified many new disease-gene associations. Yet, $\sim 50-70 \%$ of individuals with

These authors contributed equally: Alain Verloes, Lisenka E. L. M. Vissers

Members of the Solve-RD SNV-indel working group and Solve-RDDITF-ITHACA are listed below Acknowledgements.

Supplementary information The online version contains supplementary material available at https://doi.org/10.1038/s41431021-00900-2.

Charlotte W. Ockeloen

charlotte.ockeloen@radboudumc.nl

Extended author information available on the last page of the article
ID/NDD remain undiagnosed [1]. The Solve-RD project [2] systematically reanalyzes exomes and phenotypic data of 19,000 unsolved cases with rare disease from four European Reference Networks (ERNs) to elucidate the genetic etiology, including $~ 5,000$ cases from ERN-ITHACA (Intellectual Disability, TeleHealth and Congenital Anomalies; https://ern-ithaca.eu/). Exploration of mitochondrial DNA sequences extracted from WES data is part of this effort [3], as 27 of the 37 mitochondrial genes have a known diseasegene association (http://www.mitomap.org).

MT-TL1 encodes mitochondrial tRNALeu(UUR), involved in the synthesis of oxidative phosphorylation enzymes by adding leucine to the growing polypeptide chain of mtDNAencoded subunits during translation [4]. Pathogenic variants in MT-TL1 have been linked to several phenotypes associated with mitochondrial dysfunction [5], including mitochondrial myopathy, encephalopathy, lactic acidosis and stroke-like episodes (MELAS; MIM\#540000) and myoclonic epilepsy associated with ragged-red fibers (MERRF; MIM\#545000). 
We report on a variant in MT-TL1 known to interfere with mitochondrial function, uncovered by systematic reanalysis of WES data, illustrating the underexposed potential of WES-based analysis of mtDNA in identifying variants with clinical consequences.

\section{Methods}

\section{Patient inclusion}

All individuals (or legal representatives) in the Solve-RD project provided consent, compliant with local ethical guidelines and the Declaration of Helsinki. For this case, the Radboudumc Ethics Board approved the study (20184986). For publication of photos, additional consent was obtained.

\section{WES}

Diagnostic trio-based exome sequencing (proband and parents) was performed as described previously [6] using DNA isolated from whole blood.

\section{Data sharing}

Human Phenotype Ontology-coded clinical data were uploaded along with BAM files to the RD-Connect Genome-Phenome Analysis Platform (https://platform.rdconnect.eu/), and deposited at European GenomePhenome Archive (EGAZ00001527897), as part of the Solve-RD infrastructure [2]. The variant and phenotype were submitted to the Leiden Open Variation Database (individual number 00328346, phenotype number 0000246573, variant number 0000713909).

\section{Variant identification}

Systematic re-analysis of WES data is described by Matalonga et al. [3]. Details specific to this case include mapping, calling, and annotation of mtDNA using MToolBox pipeline (version 1.0) [7], with mapping against rCRS (for mtDNA) and GRCh37/Hg 19 (for genomic DNA) as reference sequences, allowing the detection of heteroplasmy levels and prioritization of variants. The following parameters were applied to identify possible diseaseassociated variants: (1) coverage $\geq 8$-fold; (2) heteroplasmy fraction $\geq 1 \%$; (3) GeneBank allele frequency (MITOMAP) $<0.2 \%$; (4) "Confirmed" or "Reported" disease association in MITOMAP; and (5) reported "Pathogenic" (ACMG, class 5) or "Likely pathogenic" (ACMG, class 4) in ClinVar.

\section{Heteroplasmy validation}

Confirmation of mitochondrial heteroplasmy was performed on blood and urine of the proband, mother and sisters using routine diagnostic procedures (PGM Ion Torrent Technology).

\section{Results}

\section{Clinical characteristics}

We report on a 23-year-old male proband with a complex neurodevelopmental and neuromuscular phenotype, who remained undiagnosed despite extensive diagnostic evaluation in a tertiary center. Family history was unremarkable, with two healthy older sisters and non-consanguineous parents. After uncomplicated pregnancy and delivery at term (normal birth weight $(3840 \mathrm{~g}$ ) and length $(52 \mathrm{~cm})$; head circumference $34 \mathrm{~cm}, 0$ SD; Apgar score 10), first concerns about development arose around 3 months of age. At age 15 months, there was severe developmental delay, consisting of hypotonia, delayed motor, social and communicative milestones, and secondary microcephaly $(44 \mathrm{~cm},-2.5 \mathrm{SD})$. Brain MRI (at 15 months; repeated at age 14 years) showed supratentorial pachygyria and frontoparietal polymicrogyria (Fig. 1A), with white matter abnormalities in the posterior limb of the internal capsule (Fig. 1B). Cerebellum and corpus callosum showed no deformities and EEG did not show epileptiform activity at age 15 months.

He developed spastic tetraparesis, orofacial dystonia, and dystonia of hands and feet. Epilepsy manifested at 7 years of age with frontal focal-onset seizures, with and without secondary tonic-clonic generalization. No myoclonus was observed. Deterioration occurred at age 17 with episodes of severe headaches, accompanied by nausea, vomiting, hematemesis, pallor and perspiration, coinciding with epileptiform activity on EEG in the left temporal lobe. Brain CT was interpreted as normal at that time (Fig. 1C). Different anti-epileptic drugs were prescribed, of which several resulted in adverse drug reactions including erythema multiforme (carbamazepine, oxcarbazepine), muscle weakness (clobazam, pregabalin), or obstructive sleep apnea syndrome (clobazam), resolving after cessation of the respective medication. Because of poor seizure control, 24-h continuous EEG monitoring was performed at 18 years and showed bilateral independent and simultaneous periodic discharges, which were more frequent during sleep than during wakefulness. He is now largely seizure free on low-dose carbamazepine. Other medical problems were severe progressive neuromuscular scoliosis (Fig. 2A), bilateral hip dysplasia 

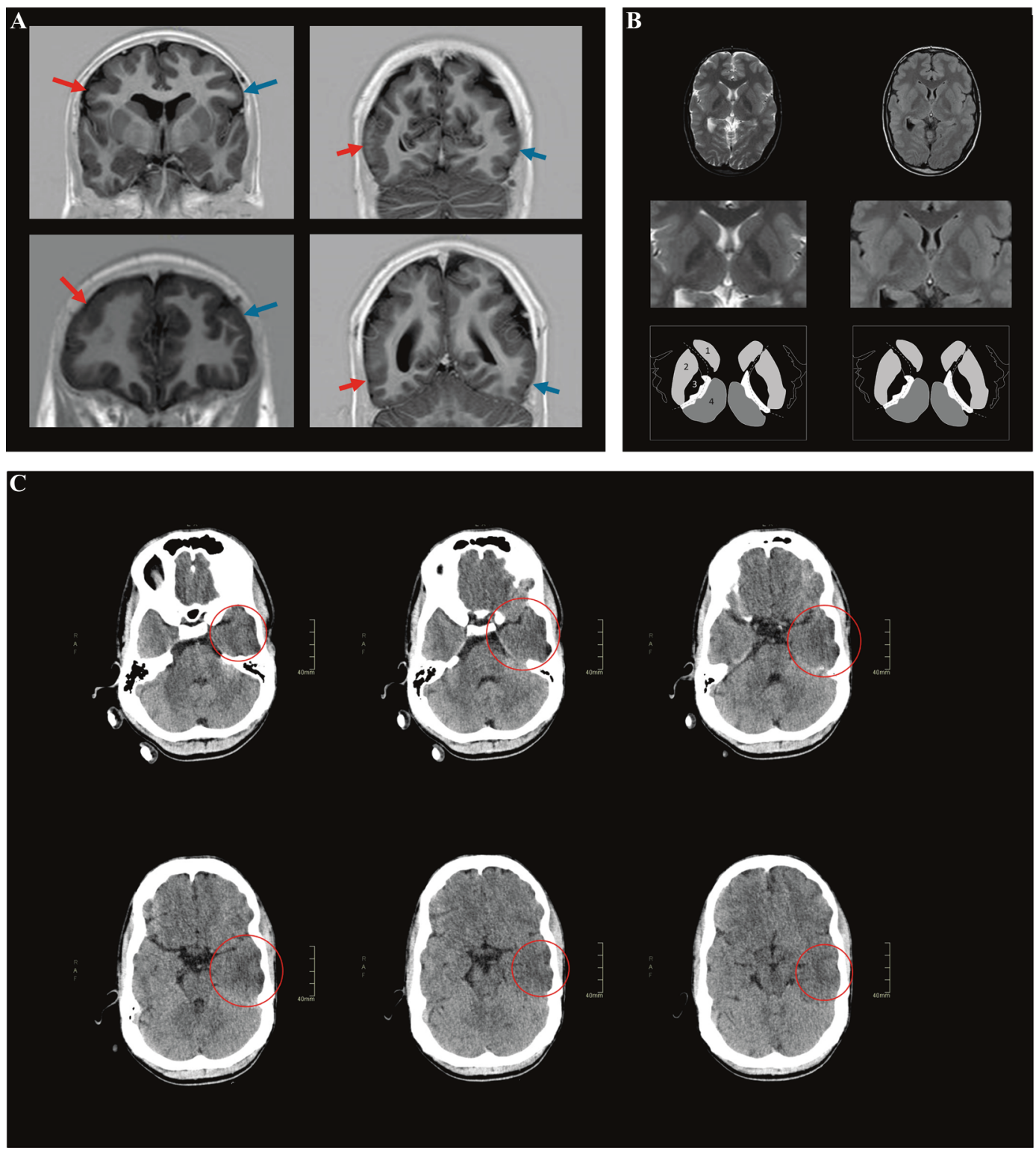

Fig. 1 Neuroimaging displays pachygyria, polymicrogyria, white matter abnormalities, and loss of gray-white differentiation. Coronal MRI images (A) in the phase-sensitive inversion recovery sequence at age 14 years. The images in the upper row show exemplary regions with a microgyrated aspect (red arrow) as compared to the contralateral region (blue arrow). The images in the lower row show exemplary regions with pachygyration (red arrow). In these regions, the gyrus-sulcus pattern is lost as compared to the contralateral side (blue arrow). Polymicrogyria and pachygyria appear most prominent in the frontoparietal cortical areas. Axial T2-weighted MR images (B; left) and fluid attenuation inversion recovery (FLAIR) images (B; right) at the level of basal ganglia at age 14 years. The middle row shows a magnification of the basal ganglia derived from images in the upper row, with a schematic representation in the lower row. 1: caudate nucleus; 2: putamen; 3 : globus pallidus; 4: thalamus; dotted line: white matter in between the basal ganglia representing the area of the internal capsule. In the posterior limb of the internal capsule, white hyperintensities are present. These can be recognized by their T2-weighted/FLAIR hyperintense aspect and suggest microstructural white matter degeneration. In addition, the globus pallidus on both sides shows a hypo-intense aspect on the T2-weighted images and FLAIR sequence. Axial CT scan images $(\mathbf{C})$ in brain tissue setting (W/L: 90/40 HU) at age 17 years. Slides should be viewed from left to right to follow the caudocranial axis; the upper-left corner shows the most caudal slide, the lower-right corner shows the most cranial slide. The red circles indicate the hypodense configuration of the temporal lobe with loss of gray-white differentiation. Gray-white differentiation refers to the appearance of the interface between cerebral white matter and cerebral gray matter on brain CT imaging. Loss of gray-white differentiation often indicates the occurrence of cytotoxic edema. In turn, cytotoxic edema is typical for infarction and hypoxicischemic encephalopathic syndromes. A clear asymmetry between the left temporal lobe and the right temporal lobe can be observed. The hypodense configuration involves the superior, middle, and inferior temporal gyri (Color figure online). 
A
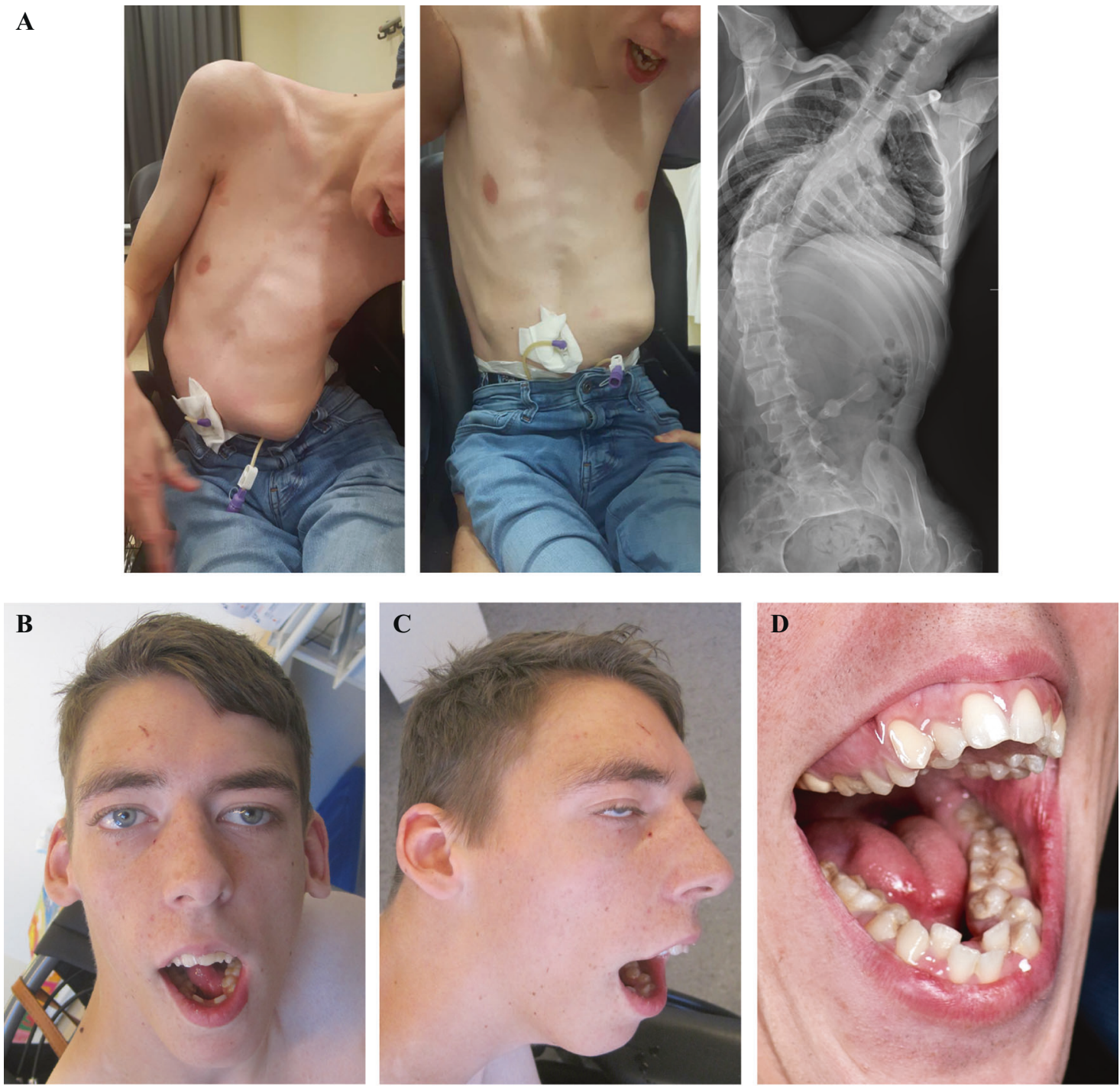

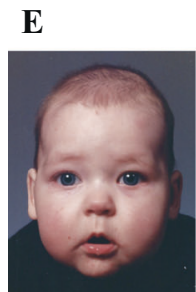

5 months

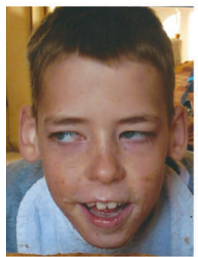

10 year

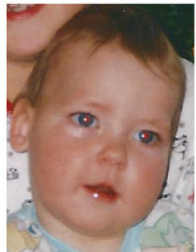

1 year

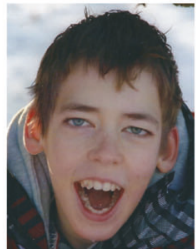

13 year

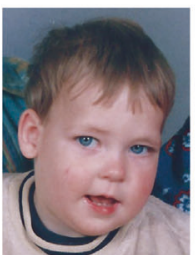

2 year

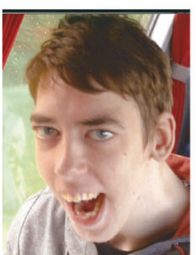

17 year

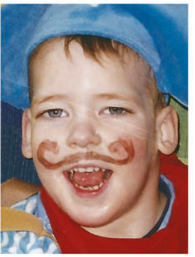

4 year

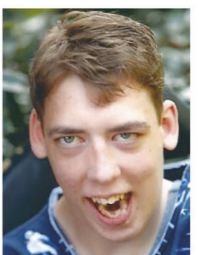

18 year

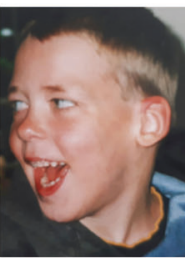

5 year

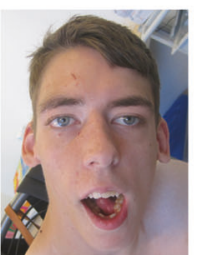

19 year

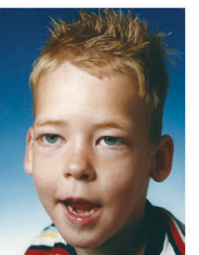

6 year

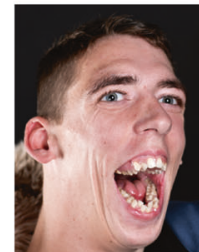

21 year

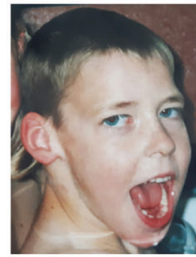

7 year

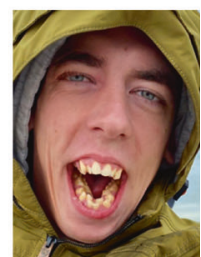

23 year with luxation, drooling with excessive tenacious stringy mucus, and severe feeding difficulties requiring gastric tube feeding and resulting in low body weight (age 18: length $165 \mathrm{~cm},-2.6 \mathrm{SD}$; weight $39.5 \mathrm{~kg}$, weight-forlength $-3.1 \mathrm{SD}$; head circumference $54 \mathrm{~cm},-1.9 \mathrm{SD}$ ). Ophthalmological assessment, visual and brainstem 
Fig. 2 Clinical and radiology images show severe scoliosis, dysmorphisms, and dental crowding. A Clinical photographs without traction (left) and with traction (middle) showing asymmetry of the chest and rib protrusion on the left side. Radiograph of the vertebral column (right) with anteroposterior view in supine position shows a slight left convex curvature of the upper thoracic spine and severe right convex scoliosis of thoracolumbar spine (Cobb's angle $\pm 75^{\circ}$ ) with axial rotation (asymmetric projection of spinous processes and pedicles) and asymmetry of the thoracic cavity. B Frontal and $\mathbf{C}$ profile facial photographs of the proband (age 19 years), showing a long face with hypotonic appearance, long palpebral fissures, a prominent nose, and small simple ears. The photograph of the mouth (D) shows crowded teeth with gingival hyperplasia (age 21 years). Facial photographs between age 5 months and age 23 years $(\mathbf{E})$, showing a progression of facial dysmorphisms with advancing age. Only mild dysmorphic features are observed in early childhood, including ptosis and a long philtrum (age 5 months to 4 years). However, the proband develops a progressively pronounced long hypotonic face with open mouth (e.g., photographs at 19 versus 10 versus 2 years of age), and has crowded teeth at age 21 (D), whereas teeth appear less crowded at earlier ages (age 7, 13, and 17 years).

auditory evoked potentials, and electrocardiography were normal. Facial dysmorphisms at 19 years of age included a long hypotonic face, full eyebrows, long palpebral fissures, a prominent nose and nasal bridge, high palate, gingival hyperplasia, and abnormal tooth implant (Fig. 2B-D), becoming more prominent over time (Fig. 2E).

Karyotype, genomic micro-array (Agilent 180k oligoarray), targeted analysis of WDR62 and ADGRG1, extensive lysosomal screening, and trio-based WES did not yield a diagnosis. Metabolic workup revealed elevation of multiple amino acids, including glycine, serine, threonine (in plasma and urine), lysine, methionine, and alanine (in plasma), in a pattern not described for a known metabolic disorder. Lactate in blood ( $1.8 \mathrm{mmol} / \mathrm{l}$, age 20 years) and cerebrospinal fluid ( $1.0 \mathrm{mmol} / \mathrm{l}$, age 15 months) were within normal range. Pharmacogenetic analysis showed a rare homozygous variant in CYP3A4 (NM_017460.5:c.878T >C;*18; rs28371759) unlikely to explain the largely dose-independent adverse drug reactions, and two rare HLA-types (HLA-A*0103; HLAB*0835) of unknown significance.

\section{Systematic re-analysis revealed a known pathogenic variant in MT-TL1}

Data of the proband and parents were included in the Solve-RD project. Prioritization of nuclear DNA variants did not yield diagnostically relevant variants, despite analysis of (de novo) variants in known disease-genes, particularly those associated with recessive or dominant cortical dysplasia, and in genes not yet implicated in NDD/ID. Variant prioritization from mtDNA revealed a variant in MT-TL1 [8], NC_012920.1:m.3291T > C (NC_012920.1:n.62T > C), known to affect mitochondrial function, at $22 \%$ heteroplasmy. The variant was absent from maternal WES data.
Validation was performed on blood of the proband and his mother using routine diagnostic procedures, displaying a heteroplasmy level of $23 \%$ in the index, compared to $4 \%$ in his mother. Urine heteroplasmy levels were $58 \%$ and $17 \%$ in index and mother, respectively. Follow-up of the family revealed heteroplasmy levels of $4 \%$ (blood) and 9\% (urine) in one of the sisters, but the variant was absent from blood and urine of the other sister. We re-evaluated brain CT imaging of the index, performed at age 17 years, which in retrospect showed early signs of stroke, including loss of gray-white differentiation in the left temporal lobe (Fig. 1C), co-localizing with epileptiform activity seen on EEG at the time of onset of episodic symptoms. Additionally, multidisciplinary evaluation and comparison of his phenotype to previously published individuals with the same variant (Table 1) revealed that the proband exhibits several symptoms seen in other individuals (epilepsy, episodic headaches with emesis, feeding difficulties, low body weight, and neuromuscular problems) [9-14], but also features that were not described before (abnormalities of brain gyration, facial dysmorphisms, early age of onset of developmental delay).

\section{Discussion}

Systematic re-analysis of existing WES data of unresolved cohorts can efficiently yield additional diagnoses [15]. Yet, re-analysis rarely includes evaluation of mtDNA although pathogenic mtDNA variants underly many rare diseases. This case illustrates the importance of including mtDNA in re-analysis, as the identification of the $M T-T L 1$ variant is of medical relevance to the proband and his sisters of childbearing age.

Mitochondrial disorders associated with mitochondrial tRNA genes are characterized by both genotypic and phenotypic heterogeneity, with poor genotype-phenotype correlations $[8,16,17]$. The m.3291T > C variant described here is located in the T-loop of mitochondrial tRNALeu(UUR). This variant was shown to result in respiratory chain enzyme deficiency and its pathogenicity was proven by single muscle fiber mtDNA analysis, showing high heteroplasmy levels in cytochrome c oxidase deficient muscle fibers [8]. Individuals carrying the m.3291T > C variant display a broad phenotypic spectrum varying from mild symptoms to severely debilitating disease [8-14, 18, 19], largely overlapping with features observed in the index. As no additional diagnostically relevant variants could be identified, it remains unclear whether the early age of onset of developmental delay, gyration defects, and dysmorphisms are attributable to a second rare genetic disorder, or expand the m.3291T >C phenotypic spectrum. However, despite gyration defects 


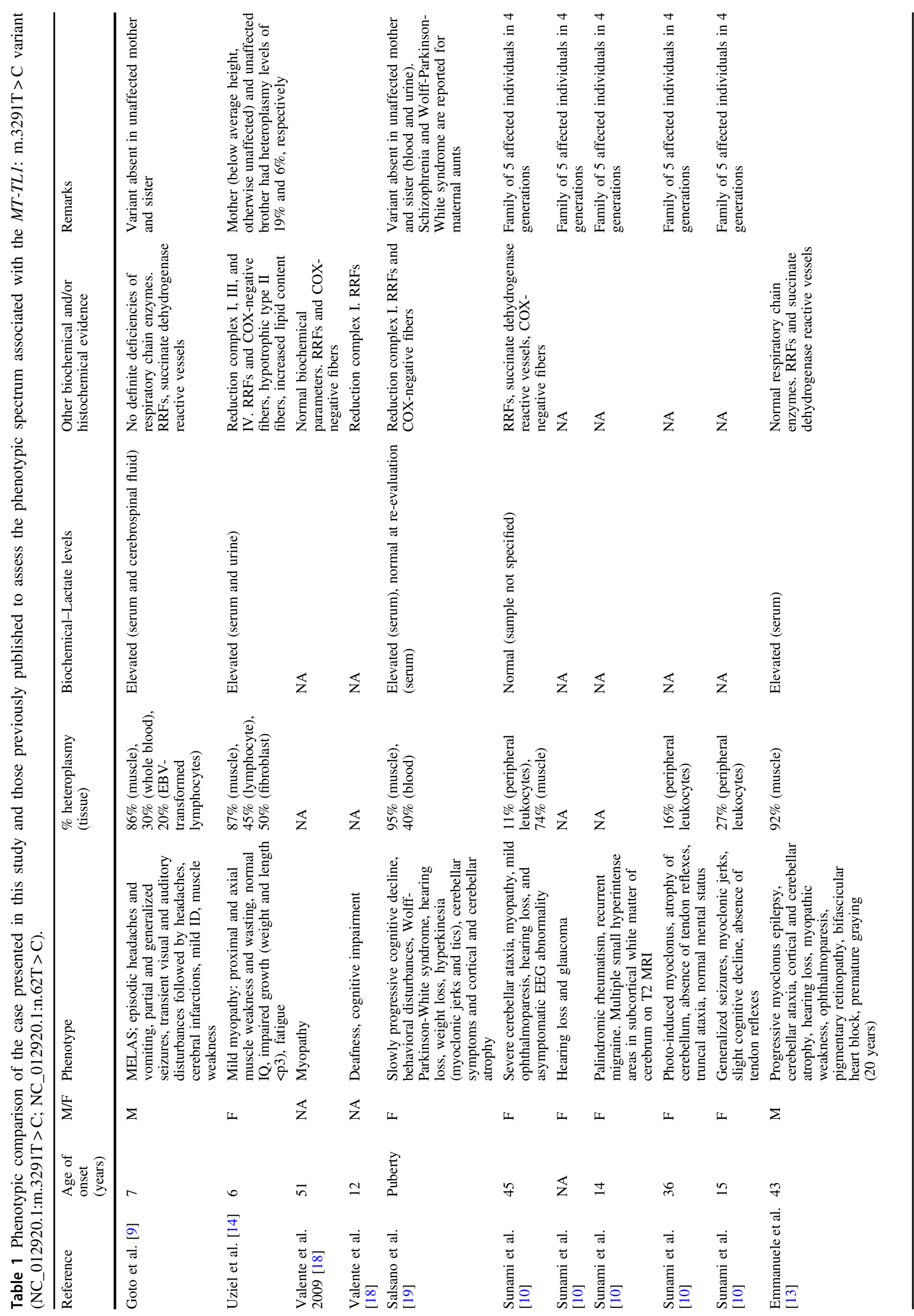




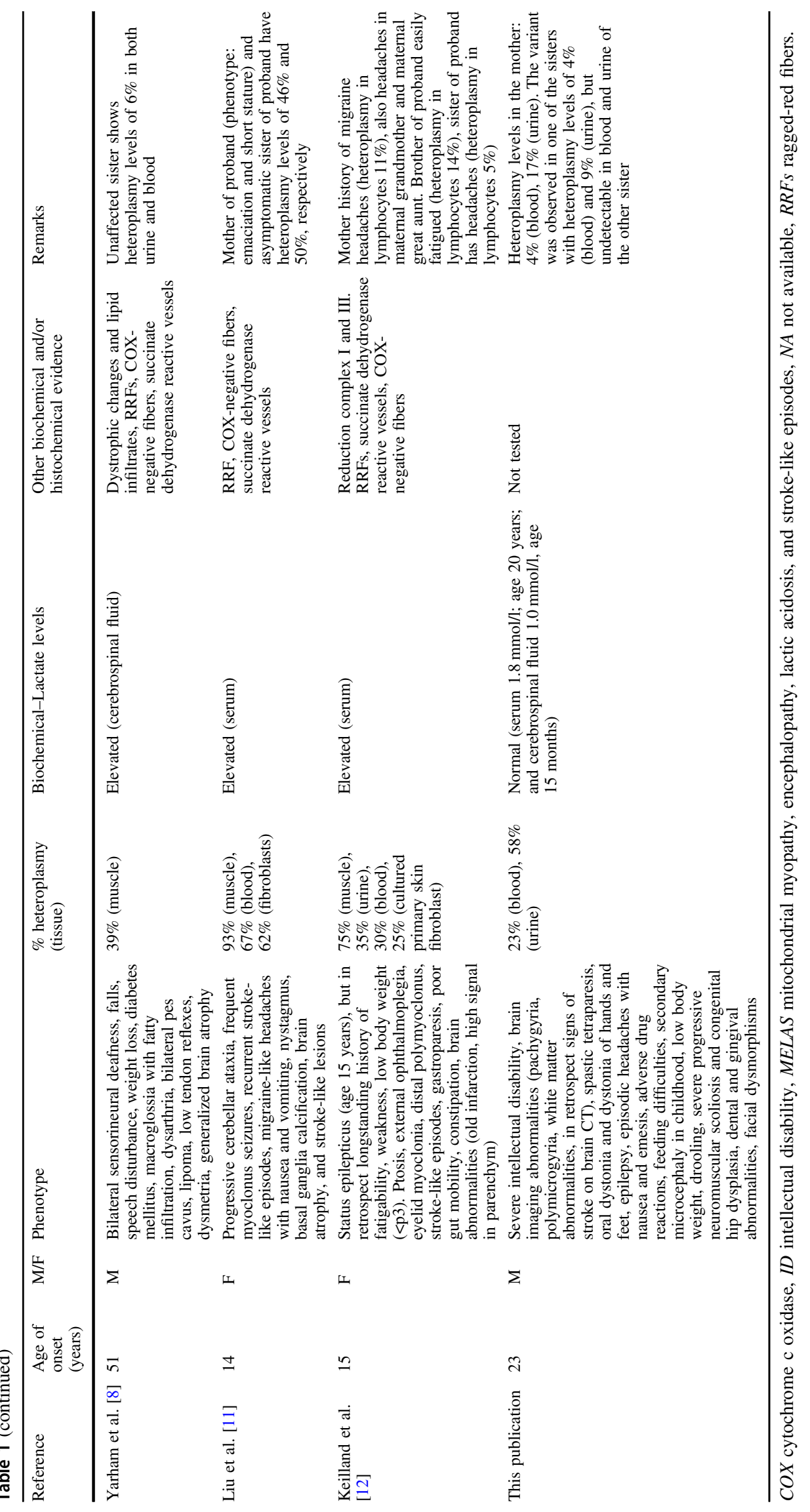


being uncommon in individuals with $M T-T L 1$ variants, polymicrogyria has been reported for $\mathrm{m} .3243 \mathrm{~A}>\mathrm{G}$ in two unrelated individuals with atypical MELAS phenotypes, additionally exhibiting other atypical symptoms also observed in the proband, including hypertonia, early onset developmental delay [20, 21] and facial dysmorphisms [20]. The proband's facial dysmorphisms might be secondary to muscle tone abnormalities. Hence, we concluded that the MT-TL1 variant could at least in part, but possibly completely, explain the proband's phenotype.

In conclusion, we describe a male proband carrying a mtDNA variant confirmed to interfere with mitochondrial function, that was identified in systematic, large-scale reanalysis on a large cohort of individuals with unresolved (syndromic) ID through the collaborative Solve-RD project. Our observations suggest that re-analysis encompassing the mtDNA interpreted from WES data may successfully yield novel unanticipated diagnoses in unexplained cases of ERN-ITHACA with implications for reproductive choices of relatives in the maternal line.

Acknowledgements We are extremely grateful to the proband and his parents for their willingness to participate in this study. In addition, we thank Michael Kwint, Kornelia Neveling, Fons Stassen, Steve Laurie, and Raul Tonda for their technical and bioinformatic support. This work has been generated through a collaboration with the European Reference Network on Rare Congenital Malformations and Rare Intellectual Disability (ERN-ITHACA) [EU Framework Partnership Agreement ID: 3HP-HP-FPA ERN-01-2016/739516]. For more information about the ERNs and the EU health strategy please visit http://ec.europa.eu/health/ern.

Solve-RD SNV-indel working group Enzo Cohen ${ }^{13}$, Isabel Cuesta ${ }^{14}$, Daniel Danis ${ }^{15}$, Anne-Sophie Denommé-Pichon ${ }^{16,17,18}$, Yannis Duffourd $^{16,18}$, Christian Gilissen ${ }^{2,19}$, Mridul Johari ${ }^{20}$, Steven Laurie ${ }^{3}$, Shuang $\mathrm{Li}^{21}$, Leslie Matalonga ${ }^{3}$, Isabelle Nelson ${ }^{13}$, Sophia Peters ${ }^{22}$, Ida Paramonov ${ }^{3}$, Sivakumar Prasanth ${ }^{23}$, Peter Robinson ${ }^{15}$, Karolis Sablauskas ${ }^{2,19}$, Marco Savarese ${ }^{20}$, Wouter Steyaert ${ }^{2,19}$, Ana Töpf ${ }^{24}$, Joeri K. van der Velde ${ }^{21}$, Antonio Vitobello ${ }^{16}$

\footnotetext{
${ }^{13}$ Sorbonne Université, INSERM UMRS_974, Center of Research in Myology, Paris, France; ${ }^{14}$ Instituto de Salud Carlos III, Madrid, Spain; ${ }^{15}$ Jackson Laboratory for Genomic Medicine, Farmington, CT, USA; ${ }^{16}$ Inserm - University of Burgundy-Franche Comté, UMR1231 GAD, Dijon, France; ${ }^{17}$ Dijon University Hospital, Genetics Department, Dijon, France; ${ }^{18}$ Dijon University Hospital, FHU-TRANSLAD, Dijon, France; ${ }^{19}$ Radboud Institute for Molecular Life Sciences, Nijmegen, The Netherlands; ${ }^{20}$ Folkhälsan Research Center, University of Helsinki, Helsinki, Finland; ${ }^{21}$ Department of Genetics, Genomics Coordination Center, University Medical Center Groningen, University of Groningen, Groningen, The Netherlands; ${ }^{22}$ Institute of Human Genetics, University of Bonn, Bonn, Germany; ${ }^{23}$ MRC Centre for Neuromuscular Diseases and National Hospital for Neurology and Neurosurgery, UCL Queen Square Institute of Neurology, London, UK; ${ }^{24} \mathrm{John}$ Walton Muscular Dystrophy Research Centre, Translational and Clinical Research Institute, Newcastle University and Newcastle Hospitals NHS Foundation Trust, Newcastle upon Tyne, UK
}

Solve-RD-DITF-ITHACA Siddharth Banka ${ }^{25,26}$, Elisa Benetti ${ }^{27}$, Giorgio Casari $^{28,29}$, Andrea Ciolfi ${ }^{30}$, Jill Clayton-Smith ${ }^{25,26}$, Bruno Dallapiccola $^{30}$, Elke de Boer ${ }^{2,31}$, Anne-Sophie Denommé-Pichon ${ }^{16,17,18}$, Kornelia Ellwanger ${ }^{32,33}$, Laurence Faivre ${ }^{16,34}$, Christian Gilissen ${ }^{2,19}$, Holm Graessner $^{32,33}$, Tobias B. Haack ${ }^{32}$, Anna Hammarsjö ${ }^{35}$, Marketa Havlovicova $^{37}$, Alexander Hoischen ${ }^{2,19,37}$, Anne Hugon ${ }^{38}$, Adam Jackson ${ }^{26}$, Tjitske Kleefstra ${ }^{2,31}$, Anna Lindstrand ${ }^{35}$, Estrella López-Martín ${ }^{39}$, Milan Macek $\mathrm{Jr}^{36}$, Leslie Matalonga ${ }^{3}$, Manuela Morleo ${ }^{29}$, Vicenzo $\mathrm{Nigro}^{29}$, Ann Nordgren ${ }^{35}$, Maria Pettersson ${ }^{35}$, Michele Pinelli ${ }^{29}$, Simone Pizzi $^{30}$, Manuel Posada ${ }^{39}$, Francesca Clementina Radio ${ }^{40}$, Alessandra Renieri $^{27,41,42}$, Caroline Rooryck ${ }^{43}$, Lukas Ryba ${ }^{36}$, Martin Schwarz ${ }^{36}$, Marco Tartaglia $^{30}$, Christel Thauvin ${ }^{16,34}$, Annalaura Torella ${ }^{28,29}$, Aurélien Trimouille $^{10,11}$, Alain Verloes ${ }^{38,44}$, Lisenka Vissers ${ }^{2,31}$, Antonio Vitobello $^{16}$, Pavel Votypka ${ }^{36}$, Klea Vyshka ${ }^{38,44}$, Birte Zurek ${ }^{32,33}$

${ }^{25}$ Manchester Centre for Genomic Medicine, St Mary's Hospital, Manchester University Hospitals NHS Foundation Trust, Health Innovation Manchester, Manchester, UK; ${ }^{26}$ Manchester Centre for Genomic Medicine, Division of Evolution and Genomic Sciences, School of Biological Sciences, Faculty of Biology, Medicine and Health, University of Manchester, Manchester, UK; ${ }^{27}$ Med Biotech Hub and Competence Center, Department of Medical Biotechnologies, University of Siena, Siena, Italy; ${ }^{28}$ Dipartimento di Medicina di Precisione, Università degli Studi della Campania "Luigi Vanvitelli,", Napoli, Italy; ${ }^{29}$ Telethon Institute of Genetics and Medicine, Pozzuoli, Italy; ${ }^{30}$ Genetics and Rare Diseases Research Division, Ospedale Pediatrico Bambino Gesù, IRCCS, Rome, Italy; ${ }^{31}$ Donders Institute for Brain, Cognition and Behaviour, Radboud University Medical Center, Nijmegen, The Netherlands; ${ }^{32}$ Institute of Medical Genetics and Applied Genomics, University of Tübingen, Tübingen, Germany; ${ }^{33}$ Centre for Rare Diseases, University of Tübingen, Tübingen, Germany; ${ }^{34}$ Dijon University Hospital, Genetics Department and Centres of Reference for Development disorders and intellectual disabilities, FHU TRANSLAD and GIMI Institute, Dijon, France; ${ }^{35}$ Karolinska Institutet, Solna, Sweden; ${ }^{36}$ Department of Biology and Medical Genetics, Charles University Prague-2nd Faculty of Medicine and University Hospital Motol, Prague, Czech Republic; ${ }^{37}$ Department of Internal Medicine and Radboud Center for Infectious Diseases (RCI), Radboud University Medical Center, Nijmegen, The Netherlands; ${ }^{38}$ Department of Genetics, Assistance Publique-Hôpitaux de Paris - Université de Paris, Robert DEBRE University Hospital, 48 bd SERURIER, Paris, France; ${ }^{39}$ Institute of Rare Diseases Research, Spanish Undiagnosed Rare Diseases Cases Program (SpainUDP) \& Undiagnosed Diseases Network International (UDNI), Instituto de Salud Carlos III, Madrid, Spain; ${ }^{40}$ Ospedale Pediatrico Bambino Gesù, Rome, Italy; ${ }^{41}$ Medical Genetics, University of Siena, Siena, Italy; ${ }^{42}$ Genetica Medica, Azienda Ospedaliero-Universitaria Senese, Siena, Italy; ${ }^{43}$ University Bordeaux, MRGM INSERM U1211, CHU de Bordeaux, Service de Génétique Médicale, Bordeaux, France; ${ }^{44}$ INSERM UMR 1141 "NeuroDiderot", Hôpital R DEBRE, Paris, France

Funding This work was financially supported by Aspasia grants of the Dutch Research Council (015.014.036 to TK and 015.014.066 to LELMV), the European Research Council (ERC to RH), the Wellcome Investigator Award (109915/Z/15/Z to RH), the Medical Research Council UK (MR/N025431/1 to RH), the Newton Fund (MR/N027302/1 to RH), the Lily Foundation (RH), and the Evelyn Trust (RH). The Solve-RD project has received funding from the European Union's Horizon 2020 research and innovation program under grant agreement No. 779257. 


\section{Compliance with ethical standards}

Conflict of interest The authors declare no competing interests.

Publisher's note Springer Nature remains neutral with regard to jurisdictional claims in published maps and institutional affiliations.

Open Access This article is licensed under a Creative Commons Attribution 4.0 International License, which permits use, sharing, adaptation, distribution and reproduction in any medium or format, as long as you give appropriate credit to the original author(s) and the source, provide a link to the Creative Commons license, and indicate if changes were made. The images or other third party material in this article are included in the article's Creative Commons license, unless indicated otherwise in a credit line to the material. If material is not included in the article's Creative Commons license and your intended use is not permitted by statutory regulation or exceeds the permitted use, you will need to obtain permission directly from the copyright holder. To view a copy of this license, visit http://creativecommons. org/licenses/by/4.0/.

\section{References}

1. Srivastava S, Love-Nichols JA, Dies KA, Ledbetter DH, Martin CL, Chung WK, et al. Meta-analysis and multidisciplinary consensus statement: exome sequencing is a first-tier clinical diagnostic test for individuals with neurodevelopmental disorders. Genet Med. 2019;21:2413-21.

2. Zurek B, Ellwanger K, Vissers LELM, Schüle R, Synofzik M, Töpf A, et al. Solve-RD: systematic Pan-European data sharing and collaborative analysis to solve Rare Diseases. Submitted to EJHG (698-20-EJHG).

3. Matalonga L, Hernández-Ferrer C, Piscia D, Vissers LELM, Schüle R, group S-RS-iw, et al. Solving patients with rare diseases through programmatic reanalysis of genome-phenome data. Submitted to EJHG (703-20-EJHG).

4. Finsterer J. Genetic, pathogenetic, and phenotypic implications of the mitochondrial A3243G tRNALeu(UUR) mutation. Acta Neurol Scand. 2007;116:1-14.

5. Chin J, Marotta R, Chiotis M, Allan EH, Collins SJ. Detection rates and phenotypic spectrum of $\mathrm{m} .3243 \mathrm{~A}>\mathrm{G}$ in the MT-TL1 gene: a molecular diagnostic laboratory perspective. Mitochondrion. 2014;17:34-41.

6. de Ligt J, Willemsen MH, van Bon BW, Kleefstra T, Yntema HG, Kroes $\mathrm{T}$, et al. Diagnostic exome sequencing in persons with severe intellectual disability. N Engl J Med. 2012;367:1921-9.

7. Calabrese C, Simone D, Diroma MA, Santorsola M, Guttà C, Gasparre G, et al. MToolBox: a highly automated pipeline for heteroplasmy annotation and prioritization analysis of human mitochondrial variants in high-throughput sequencing. Bioinformatics. 2014;30:3115-7.

8. Yarham JW, Blakely EL, Alston CL, Roberts ME, Ealing J, Pal P, et al. The m.3291T>C mt-tRNA(Leu(UUR)) mutation is definitely pathogenic and causes multisystem mitochondrial disease. J Neurol Sci. 2013;325:165-9.

9. Goto Y, Tsugane K, Tanabe Y, Nonaka I, Horai S. A new point mutation at nucleotide pair 3291 of the mitochondrial tRNA(Leu (UUR)) gene in a patient with mitochondrial myopathy, encephalopathy, lactic acidosis, and stroke-like episodes (MELAS). Biochem Biophys Res Commun. 1994;202:1624-30.

10. Sunami Y, Sugaya K, Chihara N, Goto Y, Matsubara S. Variable phenotypes in a family with mitochondrial encephalomyopathy harboring a 3291T $>C$ mutation in mitochondrial DNA. Neurol Sci. 2011;32:861-4.

11. Liu K, Zhao H, Ji K, Yan C. MERRF/MELAS overlap syndrome due to the m.3291T>C mutation. Metab Brain Dis. 2014;29:139-44.

12. Keilland E, Rupar CA, Prasad AN, Tay KY, Downie A, Prasad C. The expanding phenotype of MELAS caused by the m.3291T > C mutation in the MT-TL1 gene. Mol Genet Metab Rep. 2016;6:64-9.

13. Emmanuele V, Silvers DS, Sotiriou E, Tanji K, DiMauro S, Hirano M. MERRF and Kearns-Sayre overlap syndrome due to the mitochondrial DNA m.3291T $>$ C mutation. Muscle Nerve. 2011;44:448-51.

14. Uziel G, Carrara F, Granata T, Lamantea E, Mora M, Zeviani M. Neuromuscular syndrome associated with the 3291T $\rightarrow$ C mutation of mitochondrial DNA: a second case. Neuromuscul Disord. 2000;10:415-8.

15. Li J, Gao K, Yan H, Xiangwei W, Liu N, Wang T, et al. Reanalysis of whole exome sequencing data in patients with epilepsy and intellectual disability/mental retardation. Gene. 2019;700:168-75.

16. Yarham JW, Elson JL, Blakely EL, McFarland R, Taylor RW. Mitochondrial tRNA mutations and disease. Wiley Interdiscip Rev RNA. 2010;1:304-24.

17. Grady JP, Pickett SJ, Ng YS, Alston CL, Blakely EL, Hardy SA, et al. mtDNA heteroplasmy level and copy number indicate disease burden in m.3243A>G mitochondrial disease. EMBO Mol Med. 2018;10:e8262.

18. Valente L, Piga D, Lamantea E, Carrara F, Uziel G, Cudia P, et al. Identification of novel mutations in five patients with mitochondrial encephalomyopathy. Biochim Biophys Acta. 2009;1787:491-501.

19. Salsano E, Giovagnoli AR, Morandi L, Maccagnano C, Lamantea E, Marchesi C, et al. Mitochondrial dementia: a sporadic case of progressive cognitive and behavioral decline with hearing loss due to the rare m.3291T>C MELAS mutation. J Neurol Sci. 2011;300:165-8.

20. Keng WT, Pilz DT, Minns B, FitzPatrick DR. A3243G mitochondrial mutation associated with polymicrogyria. Dev Med Child Neurol. 2003;45:704-8.

21. Vidal A, Castillo M. Bilateral polymicrogyria and MELAS/ A3243G mutation. A very uncommon association. Neuroradiol J. 2011;24:199-201.

\section{Affiliations}

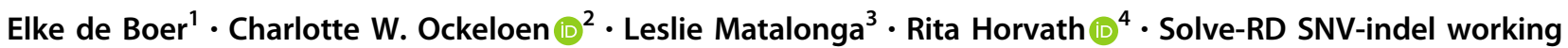

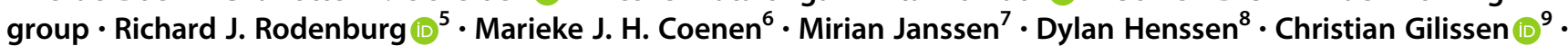 Wouter Steyaert $\mathbb{C}^{9} \cdot$ Ida Paramonov $^{3} \cdot$ Solve-RD-DITF-ITHACA . Aurélien Trimouille $\mathbb{D}^{10,11} \cdot$ Tjitske Kleefstra $^{1}$. Alain Verloes $\mathbb{D}^{12} \cdot$ Lisenka E. L. M. Vissers $\mathbb{D}^{1}$}

1 Department of Human Genetics, Donders Institute for Brain, Cognition and Behaviour, Radboud University Medical Center, Nijmegen, The Netherlands
2 Department of Human Genetics, Radboud University Medical Center, Nijmegen, The Netherlands 
3 CNAG-CRG, Centre for Genomic Regulation (CRG), The

Barcelona Institute of Science and Technology, Barcelona, Spain

4 Department of Clinical Neurosciences, University of Cambridge, John Van Geest Cambridge Centre for Brain Repair,

Cambridge, UK

5 Department of Laboratory Medicine, Translational Metabolic Laboratory, Radboud University Medical Center, Nijmegen, The Netherlands

6 Department of Human Genetics, Radboud Institute for Health Sciences, Radboud University Medical Center, Nijmegen, The Netherlands

7 Department of Internal Medicine, Radboud University Medical Center, Nijmegen, The Netherlands
8 Department of Medical Imaging, Radboud University Medical Center, Nijmegen, The Netherlands

9 Department of Human Genetics, Radboud Institute for Molecular Life Sciences, Radboud University Medical Center, Nijmegen, The Netherlands

10 Service de Génétique Médicale, Centre Hospitalier Universitaire de Bordeaux, Bordeaux, France

11 MRGM, Maladies Rares: Génétique et Métabolisme, INSERM U1211, Université de Bordeaux, Bordeaux, France

12 Département de Génétique, APHP Robert DEBRE University Hospital and INSERM U1141, Paris, France 